\title{
Beyond linear response spectroscopy of ultracold Fermi gases
}

\author{
J. Kinnunen ${ }^{\dagger}$ and P. Törmä ${ }^{\dagger *}$ \\ ${ }^{\dagger}$ Department of Physics, Nanoscience Center, P.O.Box 35, FIN-40014 University of Jyväskylä, Finland \\ * Institute for Quantum Optics and Quantum Information of the Austrian Academy of Sciences, A-6020 Innsbruck, Austria
}

We study RF-spectroscopy of ultracold Fermi gas by going beyond the linear response in the field-matter interaction. Higher order perturbation theory allows virtual processes and energy conservation beyond the single particle level. We formulate an effective higher order theory which agrees quantitatively with experiments on the pairing gap, and is consistent with the absence of the mean-field shift in the spin-flip experiment.

A single atom interacting with a coherent field displays various coherent phenomena, such as Rabi oscillations, which can be theoretically described by the exact nonperturbative solution of the quantum time evolution of the system [1]. In many other contexts, however, it is sufficient to treat the field-matter interaction within the linear response, i.e. to first order in perturbation theory; this allows one, for instance, to calculate the response of matter in a complicated many-body state [2]. In ultracold Fermi gases of alkali atoms, the interaction between atoms and fields may be extremely coherent while, at the same time, the atoms can be strongly interacting or in a non-trivial many-body state such as superfluid of Cooper pairs. This is likely to lead to new phenomena in the field-matter interactions. The first indication of this was the absence of mean-field shifts observed [3] in the spin-flip experiment using RF fields [4,3]. The experimental results were in contradiction with predictions given by linear response. However, they were elegantly explained [3, 5] as a collective, coherent rotation of the Bloch vector within a framework that can be applied to Hartree-type mean-field interactions. It has now become topical to ask what happens in a similar situation but for a more complicated many-body state, such as the recently observed Fermi superfluids [6]. RF-spectroscopy was used for detecting the pairing gap in such systems 71. The results were qualitatively in good agreement [8, 9, 10. with the lowest order perturbation theory.

In this letter we combine higher order perturbative approach of the field-atom interaction with the idea of a collective coherent rotation of all atoms [5] and apply it to the scheme used in the experiment [7]. The key point is that only total energy conservation is demanded instead of energy conservation in each single-atom process. This approach allows the transfer of large numbers of atoms, as observed in the experiment [7] and is consistent with the absence of mean-field shifts in the spin-flip experiment [5]. It is a step towards high precision description of the RF-spectroscopy of superfluid Fermi gases, and it highlights that ultracold Fermi gases may display new phenomena in field-matter interactions.

We study the RF-spectroscopy of a trapped Fermi gas, as described in Fig. 1] using perturbative expansion of the field-atom coupling. The interaction is described in the rotating wave approximation, for a spatially constant field with the Rabi frequency $\Omega$, by

$$
H_{\mathrm{I}}=\Omega \int d \mathbf{r}\left[e^{i \omega t} \Psi_{e}^{\dagger}(\mathbf{r}) \Psi_{g}(\mathbf{r})+h . c .\right]
$$

where $\omega$ is the detuning of the RF-field and the field operators are expanded in the eigenstates of the harmonic trap potential $\Psi_{\sigma}(\mathbf{r})=\sum_{n l m} \psi_{n l m}(\mathbf{r}) c_{n l m \sigma}$. In the following, the term "momentum conservation" should be understood, in case of a nonuniform gas, as the conservation of the trap quantum number.

The lowest order perturbation theory in $H_{\mathrm{I}}$ using the exact trap states gives the transfer probability $P_{i}=$ $\int d \mathbf{r} \int d \mathbf{r}^{\prime} P\left(i, \mathbf{r}, \mathbf{r}^{\prime}\right)$ for atom $i=\left(n_{i}, l_{i}, m_{i}\right)$. Here

$$
\begin{aligned}
P\left(i, \mathbf{r}, \mathbf{r}^{\prime}\right)= & \frac{\Omega^{2}}{\hbar^{2}} \int_{0}^{t} d \tau_{1} \int_{0}^{t} d t_{1} e^{i \omega\left(t_{1}-\tau_{1}\right)} \sum_{j j^{\prime}} \Psi_{i j j^{\prime}}\left(\mathbf{r}, \mathbf{r}^{\prime}\right) \\
& \times\left\langle c_{i g}^{\dagger}\left(\tau_{1}\right) c_{j g}\left(t_{1}\right)\right\rangle\left\langle c_{j^{\prime} e}\left(\tau_{1}\right) c_{j^{\prime} e}^{\dagger}\left(t_{1}\right)\right\rangle,
\end{aligned}
$$

where the function $\Psi_{i j j^{\prime}}\left(\mathbf{r}, \mathbf{r}^{\prime}\right)=\psi_{i}(\mathbf{r}) \psi_{j^{\prime}}(\mathbf{r}) \psi_{j}\left(\mathbf{r}^{\prime}\right) \psi_{j^{\prime}}\left(\mathbf{r}^{\prime}\right)$ describes the overlap of atom wavefunctions. The expectation value for the number of transferred atoms in the first-order perturbation theory is obtained by summing Eq.(2) over all atom indices $i=\left(n_{i}, l_{i}, m_{i}\right)$. In addition, the integration over the times $\tau_{1}$ and $t_{1}$ gives the energy conservation condition for a single-atom process. We consider the term of the order $N$ in the perturbation expansion, (c.f. Fig. 21)

$$
\left\langle\hat{N}_{e}(t)\right\rangle_{N}=\frac{1}{\hbar^{2 N}}\left\langle\int_{0}^{t} d t_{1} \ldots \int_{0}^{t_{N-1}} d t_{N} H_{I}\left(t_{N}\right) \ldots H_{I}\left(t_{1}\right) N_{e}(t) \int_{0}^{t} d \tau_{N} \ldots \int_{0}^{\tau_{2}} d \tau_{1} H_{I}\left(\tau_{N}\right) \ldots H_{I}\left(\tau_{1}\right)\right\rangle
$$




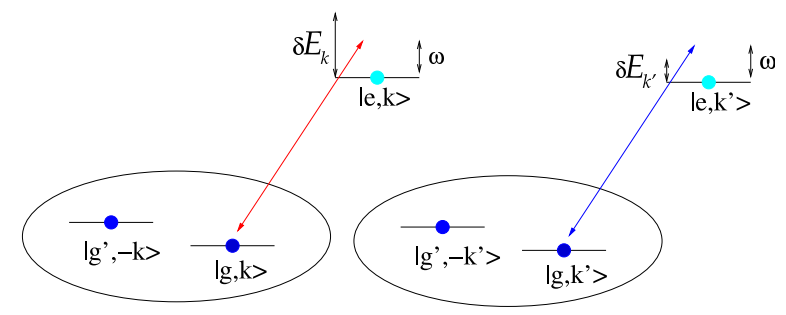

Figure 1: The RF-field breaks a pair of atoms in internal states $g^{\prime}$ and $g$ by driving a transition to a third state. At the single-atom level, the energy cost of creating an excitation in the superfluid, $\delta E_{k}$, has to match the RF-field detuning $\omega$, causing a shift of the spectral peak. Higher order perturbation theory allows processes where energy is conserved at the many particle level, e.g. resonant transfer of the two atoms above even when they are individually out of resonance.

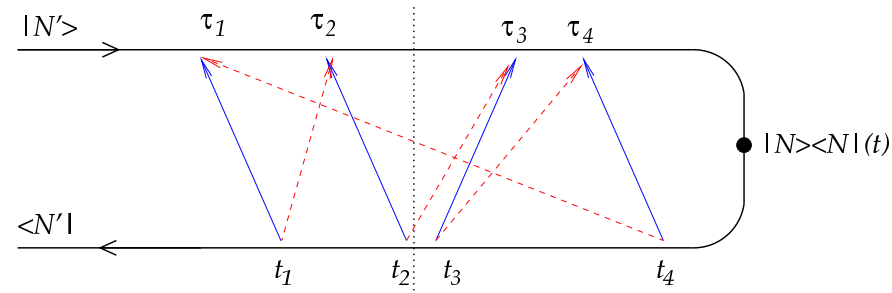

Figure 2: In the sequential transfer (solid arrows) of $N=4$ atoms, the (time)order in which the atoms are transferred can be chosen in $N$ ! different ways. An $N$-atom transfer (one example shown by dashed arrows) can be done in $N !^{2}$ different ways. The process is called virtual if an auxiliary vertical line (example shown as dotted line) cuts two or more arrows 11].

We take this to be the expectation value for $N$ atoms being transferred in a single process. Thus, we neglect oscillations of the atoms back and forth corresponding to terms of higher order in $H_{I}$ but with the same final number of transferred atoms. In principle, these oscillations contribute to the linewidth of the field-atom interaction.

For weak excitations (linear response), the singleparticle energy conservation gives the transfer probability $\frac{\Gamma^{2}}{\left(\delta E_{k}-\omega\right)^{2}+\Gamma^{2}}$ for an atom with energy cost $\delta E_{k}$, where $\omega$ is the field detuning and $\Gamma$ is the linewidth. For strong single-particle excitations, i.e. Rabi oscillations, this becomes $\frac{\Omega^{2}}{\left(\delta E_{k}-\omega\right)^{2}+\Omega^{2}}$, thereby the field-atom coupling $\Omega$ acts as an effective linewidth. Large effective linewidth $(\Gamma$ or $\Omega$ ) allows to transfer a large number of atoms nonresonantly also in single-particle processes. However, the reported linewidth in [7] is small, $\sim 100 \mathrm{~Hz}$, which could also be deduced from the sharpness of the narrowest features of the spectra, giving a limit to homogenous broadening (other features show inhomegenous broadening due to pairing and the trap potential). The effective linewidth needed for the transfer of the observed number of atoms can be estimated, using the calculated dispersion $\delta E_{k}$, to be $\sim 10 \mathrm{kHz}$. Since this is significantly larger than $100 \mathrm{~Hz}$, considerations beyond single-particle processes are well motivated.

Assume an effective pulse length $T_{\mathrm{P}}$ (either the true pulse length or the coherence time of the interaction). If the number of transferred atoms $N$ is large, the transfer processes occur at roughly even intervals. This means that each intermediate virtual state lasts for roughly the same time $t_{\text {inter }}=\frac{T_{\mathrm{P}}}{N-1}$. For pulse lengths of $T_{\mathrm{P}}=1 \mathrm{~s}$, and the number of transferred atoms of the order $N=$ $10^{5}$ the corresponding energy uncertainty for any single virtual state is $\Delta E / \hbar \geq \frac{1}{t_{\text {inter }}}=10^{5} \mathrm{~Hz}$ (repeated transfer of smaller $N$ within coherence-limited $T_{\mathrm{P}}$ gives the same result). Since the typical Fermi energies are of the order of $10 \mathrm{kHz}$, this allows any atom to be transferred regardless of its energy cost. However, the total Nth order process should conserve energy, meaning that the sum of $\delta E_{k}-\omega$ of all transferred atoms should be zero.

The effective pulse length $T_{\mathrm{P}}$ enters the picture only through the uncertainty relation, by introducing an energy cutoff $\delta_{\mathrm{c}}$ for the maximum energy cost of an atom transferred through an intermediate (virtual) state. This allows to simplify Eq. (3) by fixing the cutoff $\delta_{\mathrm{c}}$. Thereby the actual duration of the virtual state $t_{\text {inter }}$ becomes irrelevant for $t_{\text {inter }} \leq \frac{\hbar}{\delta_{\mathrm{c}}}$ and we can choose $t_{\text {inter }} \rightarrow 0$. Considering the RF-spectroscopy in the sense of a local density approximation, calculating the spectra for each position $\mathbf{r}$ separately and then integrating over $\mathbf{r}$, we also demand that the atoms may not exchange energy in the Nth order process over a distance. The probability of finding the atoms at the same position is given by the overlap of the atom wavefunctions. These approximations can be written as

$$
\left\langle\hat{N}_{e}(t)\right\rangle_{N} \approx \frac{t^{2 N-2}}{\hbar^{2 N}} \int_{0}^{t} d \tau_{1} \int_{0}^{t} d t_{1}\left\langle H_{I}^{N}\left(\tau_{1}\right) N_{e}(t) H_{I}^{N}\left(t_{1}\right)\right\rangle
$$

where

$$
H_{I}^{N}(t)=C \int d \mathbf{r}\left[\Omega e^{i \omega t} \Psi_{e}^{\dagger}(\mathbf{r}, t) \Psi_{g}(\mathbf{r}, t)+h . c .\right]^{N},
$$

and the normalisation constant $C=V^{N-1}$. The integration over the position $\mathbf{r}$ gives the total momentum conservation. Having only two time variables in Eq.(4) is related to the simultaneous transfer approximation $\left(t_{\text {inter }} \rightarrow 0\right)$, and the single position variable in Eq.(15) corresponds to the requirement of the wavefunction overlap. For fermions, the operator $H_{I}^{N}$ as expressed above vanishes for $N>1$ because of the Pauli exclusion principle. However, restricting oneself to a subset where each one-atom transfer preserves momentum separately, one obtains a nonvanishing contribution even for fermions. That is, we replace

$$
\Psi_{e}^{\dagger}(\mathbf{r}, t) \Psi_{g}(\mathbf{r}, t) \rightarrow \sum_{n l m}\left|\psi_{n l m}(\mathbf{r})\right|^{2} c_{n l m e}^{\dagger}(t) c_{n l m g}(t) .
$$

The process described here is therefore only possible in presence of momentum conservation in the field-matter 
interaction, as is indeed the case in the considered system.

Our Nth order theory is now simply a linear response theory for the defined coherent $\mathrm{N}$-atom coupling operator $H_{I}^{N}(t)$. One obtains using the Wick's theorem (when no atoms in state $e$ initially)

$$
\left\langle\hat{N}_{e}\left(t, \mathbf{r}, \mathbf{r}^{\prime}\right)\right\rangle_{N}=C^{\prime} \int_{0}^{t} d \tau_{1} \int_{0}^{t} d t_{1} e^{i N \omega\left(t_{1}-\tau_{1}\right)} \sum_{\{i\}} P_{\{i\}}
$$

where the prefactor $C^{\prime}=\frac{N C^{2} t^{2 N-2} \Omega^{2 N}}{\hbar^{2 N}}$, the summation is over all $N$-atom sets $\{i\}$ and the probability that this set of atoms $\{i\}$ is transferred is

$$
P_{\{i\}}=\sum_{\overline{\{j\}}} \prod_{i, j \leq N} \Psi_{i j}\left(\mathbf{r}, \mathbf{r}^{\prime}\right)\left\langle c_{i g}^{\dagger}\left(\tau_{1}\right) c_{j g}\left(t_{1}\right)\right\rangle\left\langle c_{i e}\left(\tau_{1}\right) c_{i e}^{\dagger}\left(t_{1}\right)\right\rangle .
$$

Here $\overline{\{j\}}$ denotes all permutations of the set $\{i\}$. Note that even though each one-atom process preserves momentum, the Nth order process still contains off-diagonal Green's functions $\left\langle c_{n l m g}^{\dagger} c_{n^{\prime} l^{\prime} m^{\prime} g}\right\rangle$.

We approximate the sum over all permutations $\overline{\{j\}}$ by $N$ independent sums, thus replacing the $N$ ! terms by $N^{N}$ terms. This approximation contains all the correct terms but it also introduces additional terms. However, the diagonal Green's functions dominate over the off-diagonal ones, and these additional terms give only a small contribution. In addition, the product over the functions $\Psi_{i j}$ gives cancellations of the added terms upon $\mathbf{r}$-integration since, for these terms, the atom wavefunctions do not necessarily appear in the square form $\left|\Psi_{i j}\right|^{2}$. One obtains the following form for the transfer probability of set $\{i\}$

$$
P_{\{i\}}=\prod_{i \leq N} \sum_{j} \Psi_{i j}\left(\mathbf{r}, \mathbf{r}^{\prime}\right)\left\langle c_{i g}^{\dagger}\left(\tau_{1}\right) c_{j g}\left(t_{1}\right)\right\rangle\left\langle c_{i e}\left(\tau_{1}\right) c_{i e}^{\dagger}\left(t_{1}\right)\right\rangle .
$$

This probability can be formally written at $T=0$ as

$$
P_{\{i\}}=\prod_{i \leq N} \sum_{j} \sum_{m} e^{i \Delta E(i, j, m)\left(\tau_{1}-t_{1}\right)} F\left(i, j, m, \mathbf{r}, \mathbf{r}^{\prime}\right),
$$

where $\Delta E(i, j, m)$ is the energy difference of the singleatom states and $F(i, j, m)$ is a product of Fermi functions and Bogoliubov coefficients giving the occupation and transfer probabilities, irrespective of the energy conservation. The internal variable $m$, describing the quasiparticle states, comes from the Bogoliubov-deGennes equations. Comparison of Eq.(9) to Eq.(2) shows that the first-order term contains the same spectral weight factors, if we choose $j=j^{\prime}$ in Eq. (2). The sum in Eq. (2) becomes

$$
\sum_{j} \sum_{m} e^{i \Delta E(i, j, m)\left(\tau_{1}-t_{1}\right)} F\left(i, j, m, \mathbf{r}, \mathbf{r}^{\prime}\right) .
$$

Integration over the times $\tau_{1}$ and $t_{1}$ gives the single atom energy conservation. In (10), the exponential functions in the product over $i$ can be combined and upon integration over times $\tau_{1}$ and $t_{1}$ in Eq.(4), yield the total energy conservation. This acts as a boundary condition for the choice of the transferable atoms $\left\{\left(n_{i}, l_{i}, m_{i}\right)\right\}$ and hence for the actual number of transferred atoms $N$. The expectation number of transferred atoms is now the average of all combinations $\{\mathcal{A}\}$ that satisfy the total energy conservation. The spectral weights, or the probabilities, of different combinations are determined by the product of the terms $F\left(i, j, m, \mathbf{r}, \mathbf{r}^{\prime}\right)$ which also appear in the first order spectra in Eq. (11). We approximate the expectation value by keeping in $\{\mathcal{A}\}$ only the combination that transfers the highest number of atoms. We have tested this approximation with 40 atoms and found good agreement between the approximative scheme and the full combinatorial treatment. In calculating the total energy conservation, the energy cutoff $\delta_{\mathrm{c}}$ for the maximum energy cost of an transferrable atom acts as an additional constraint for the possible combinations.

We now use the first order spectrum to calculate the number of transferred atoms by the following algorithm.

- Consider a fixed field detuning $\omega$. Set $\delta_{\min }=-\infty$, $\delta_{\max }=\infty$. Let $N_{1}(\delta)$ be the expectation number of atoms that one can transfer using the first-order perturbation theory and by giving energy $\delta$.

- Integrate $\int_{\delta_{\min }}^{\delta^{\max }} d \delta N_{1}(\delta)(\delta \quad-\quad \omega) \quad=$ : $\Delta E\left(\delta_{\min }, \delta_{\max }, \omega\right)$. This gives the total energy change if all the atoms with energy change of $\delta \in\left[\delta_{\text {min }}, \delta_{\text {max }}\right]$ are transferred.

- If $\Delta E\left(\delta_{\min }, \delta_{\max }, \omega\right)$ is positive, decrease $\delta_{\max }$ or if it is negative, increase $\delta_{\min }$ and go back to the previous step. Otherwise, proceed to the next step.

- The maximum number of atoms that one can transfer is $N_{N}(\omega):=\int_{\delta_{\min }}^{\delta_{\max }} d \delta N_{1}(\delta)$.

One can map the whole spectrum by using the algorithm above for several detunings $\omega$. For a nonuniform gas, the process should be repeated for all positions $\mathbf{r}$ and $\mathbf{r}^{\prime}$ and integrated to give the total number of transferred atoms.

For a noninteracting gas, the first-order perturbation theory and the current higher order theory give identical results since the conservation of the total energy equals the conservation of energy of a single one-atom process. For atoms interacting only via the Hartree field, the two theories differ by the position of the spectral peak. As an example we consider the spin-flip experiment of Ref. [5], with $N_{g}\left(N_{g^{\prime}}\right)$ atoms in the $g$-state $\left(g^{\prime}\right.$-state) and mutual interaction energy $V_{g g^{\prime}}$. In the first-order perturbation theory, the RF-field needs to give the energy corresponding to a single atom excitation, i.e. $\Delta E=V_{g g^{\prime}}\left(N_{g}-N_{g^{\prime}}\right)$. The higher order theory requires only the energy corresponding to the complete $N$-atom process. Since the mutual interaction energy of the two fermion species $g$ 
and $g^{\prime}$ is unchanged if the spins of all atoms are flipped, the total energy change of the whole process is zero 12 . This is consistent with the absence of the mean-field shift observed in the spin-flip experiment in Ref. [5].

To describe the superfluid Fermi gas in a harmonic trap, we apply the mean-field resonance superfluidity approach used in Ref. [9] but restricting oneself to the one-channel model in order to describe broad Feshbachresonances [13, 14]. We calculate self-consistently the position dependent excitation gap, density distribution and the chemical potential for 2670 atoms using background interaction strength $U=2.3 \hbar \omega_{0} r_{\text {osc }}^{3}$ and cutoff $E_{c}=161.5 \hbar \omega_{0}$, where $\hbar \omega_{0}, r_{\text {osc }}$ are the oscillator energy and the length. The position dependent excitation gap is $0.83 E_{\mathrm{F}}$ at the center of the trap corresponding roughly to $\mathrm{Li}^{6}$ atoms with peak density of $10^{13} 1 / \mathrm{cm}^{3}$ at the magnetic field of $B \approx 834 \mathrm{G}$, as obtained from the renormalised theory for a uniform gas [8]. The small number of atoms used above gives too narrow density and gap profiles, and one expects a larger excitation gap close to the edges of the trap for numbers of atoms used in the experiments. On the other hand, for pulses longer than the coherence time of the atoms, the system can relax and lower the excitation gap during the pulse (not considered here). Thus, one expects the two approximations to partly cancel each other.

Fig. 3 shows the RF-spectra at two temperatures $T=$ $0.0 T_{\mathrm{F}}$ and $0.08 T_{\mathrm{F}}$ using a $\pi / 2$-pulse. The peak is shifted due to the pairing gap and, most notably, another peak at the zero detuning exists at a finite temperature, originating from contributions from the edges of the trap [8]. This behaviour and the locations of the two peaks at finite temperatures are in good quantitative agreement with the experiment [7]. Note that, in principle, firstorder perturbation theory gives a maximum transfer ratio of only $\sim 5 \%$ of the atoms, because the single particle excitation energies $\delta E_{k}$ have a strong momentum $(k)$ dependence and therefore only a small fraction of the atoms can match the energy of a narrow linewidth field. The higher order theory is not limited in this way and allows to describe the experiments with large numbers of transferred atoms, such as $50 \%$ in [7]; c.f. Fig. [3

In conclusion, the connection between the first-order and higher order terms in perturbation theory was used to define an effective higher order theory for the RFspectroscopy of Fermi gases. It was shown to be consistent with the temperature dependence of the pairing gap spectra 7] and the coherence phenomenon observed in Ref. [5]. This approach could be applied also to other types of field-matter interactions and spectroscopies in ultracold gases. It should be useful whenever the single particle excitation spectrum is non-trivial, e.g. BCS-type states or exotic states in optical lattices, and the process is coherent. Due to the special nature of field-matter interactions discussed here, the understanding of phenomena such as Josephson oscillations in Fermi gases - when

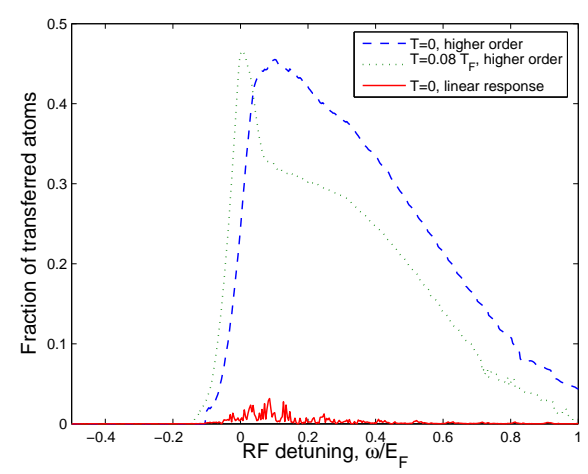

Figure 3: RF-spectra in the higher order theory using parameters given in the text. At finite temperatures, an additional peak appears at the zero detuning. For comparison, the spectrum from the linear response theory is shown in solid line.

the effective tunneling is realized by fields 15 - will be non-trivial and may show phenomena not existent in the corresponding solid state system.

Acknowledgements P.T. thanks the Aspen Center for Physics. This project was supported by Academy of Finland and EUROHORCs (EURYI award, Academy project numbers 106299, 205470), and the QUPRODIS project of EU.

[1] C. Cohen-Tannoudji, J. Dupont-Roc, and G. Grynberg, Atom-Photon Interactions (Wiley, New York, 1998).

[2] G. D. Mahan, Many-Particle Physics (Plenum Publishers, New York, 2000).

[3] S. Gupta et al., Science 300, 1723 (2003).

[4] C. A. Regal and D. S. Jin, Phys. Rev. Lett. 90, 230404 (2003).

[5] M. W. Zwierlein et al., Phys. Rev. Lett. 91, 250404 (2003).

[6] For references see the review R. Grimm, Nature 435, 1035 (2005).

[7] C. Chin et al., Science 305, 1128 (2004).

[8] J. Kinnunen, M. Rodríguez and P. Törmä, Science 305, 1131 (2004).

[9] Y. Ohashi and A. Griffin, Phys. Rev. A 72, 013601 (2005).

[10] Y. He, Q. Chen, and K. Levin, Phys. Rev. A 72, 011602(R) (2005).

[11] H. Schoeller and G. Schön, Phys. Rev. B 50, 18436 (1994).

[12] It is always this energy change that appears in the exponential terms in Eq. (10). How many spins are actually flipped depends on the factor in front, $\Omega t$, i.e. whether $\pi / 2$ pulse or shorter is applied, but the energy term contains the final energy corresponding to all spins flipped.

[13] G. M. Bruun et al., Eur. Phys. J. D 9, 433 (1999).

[14] Y. Ohashi, and A. Griffin, cond-mat/0508213

[15] Gh.-S. Paraoanu, M. Rodriguez, and P. Törmä, Phys. Rev. A 66, 041603(R) (2002). 